\title{
Dinesh Tripathi
}

\section{A note on Eneström-Kakeya theorem for a polynomial with quaternionic variable}

Received: 6 June 2019 / Accepted: 15 April 2020 / Published online: 12 May 2020

(C) The Author(s) 2020

\begin{abstract}
In this paper, we present certain results concerning the location of the zeros of polynomials with quaternionic variable which generalize and refine some known Eneström-Kakeya type bounds for the zeros of polynomials.
\end{abstract}

\section{Mathematics Subject Classification 30E10; 30A01 - 30C01}

\section{Introduction}

Let $p(z)=\sum_{j=0}^{n} a_{j} z^{j}$ be a polynomial of degree $n$. Then, concerning the distribution of complex zeros of polynomial $p(z)$, Eneström and Kakeya $[3,8]$ proved the following result.

Theorem 1.1 All the complex zeros of polynomial $p(z)=\sum_{l=0}^{n} a_{l} z^{l}$ of degree $n$ with real coefficients $a_{l}, 0 \leq$ $l \leq n$, such that $a_{n} \geq a_{n-1} \geq \cdots \geq a_{1} \geq a_{0}>0$ lie in $|z| \leq 1$.

In the literature [1-4,7-10], there exist several extensions and generalizations of Theorem 1.1. Joyal et al. [9] extended Theorem 1.1 by removing non-negative restriction over the coefficients of polynomial $p(z)$. In fact, they proved the following result.

Theorem 1.2 All the complex zeros of the polynomial $p(z)=\sum_{l=0}^{n} a_{l} z^{l}$ of degree $n$ with real coefficients, such that $a_{n} \geq a_{n-1} \geq \cdots \geq a_{1} \geq a_{0}$, lie in disk:

$$
|z| \leq \frac{1}{\left|a_{n}\right|}\left(\left|a_{n}\right|-a_{0}+\left|a_{0}\right|\right) .
$$

In this paper, we will prove some extensions and generalizations of Theorems 1.1 and 1.2 for the class of polynomials with quaternionic variable and quaternionic coefficients.

\section{Background}

The quaternions are a number system that extends the complex numbers. That was first described by Irish mathematician Sir William Rowan Hamilton in 1843 and applied to mechanics in three-dimensional space. The set of quaternions are denoted by $\mathbb{H}$ in the honour of Sir Hamilton. A feature of quaternions is that multiplication of two quaternions is noncommutative. Quaternions are generally represented in the form:

D. Tripathi $(\varangle)$

Department of Mathematics, Faculty of Applied Science, Manav Rachna University, Faridabad 121004, India

E-mail: dinesh@mru.edu.in; dineshtripathi786@gmail.com 
$q=\alpha+i \beta+j \gamma+k \delta \in \mathbb{H}$, where $\alpha, \beta, \gamma, \delta \in \mathbb{R}$, and $i, j$, and $k$ are the fundamental quaternion units, such that $i^{2}=j^{2}=k^{2}=i j k=-1$.

Let

$$
\mathbb{P}_{n}:=\left\{p ; \quad p(q)=\sum_{l=0}^{n} q^{l} a_{l}, \quad q \in \mathbb{H}\right\}
$$

denote the class of $n^{t h}$-degree polynomials with quaternionic variable $q \in \mathbb{H}$ and $a_{l}, 0 \leq l \leq n$ are either real or quaternion.

Recently, Carney et al. [2] proved the following extension of Theorem 1.1 for the polynomial $p(q)$.

Theorem 2.1 All the zeros of the polynomial $p \in \mathbb{P}_{n}$ of degree $n$ with real coefficients, such that $a_{n} \geq a_{n-1} \geq$ $\cdots \geq a_{1} \geq a_{0}>0$ lie in $|q| \leq 1$.

In the same paper, they proved the following refinement of Theorem 2.1 by removing the positivity restriction on the coefficients of $p(q)$. This gives a generalization of Theorem 1.2 for $p \in \mathbb{P}_{n}$ with quaternions $a=$ $\alpha+i \beta+j \gamma+k \delta \in \mathbb{H}$.

Theorem 2.2 All the zeros of the polynomial $p \in \mathbb{P}_{n}$ of degree $n$ with quaternionic coefficients $a_{l} \in \mathbb{H}, 0 \leq$ $l \leq n$ such that $\alpha_{n} \geq \alpha_{n-1} \geq \cdots \geq \alpha_{1} \geq \alpha_{0}, \beta_{n} \geq \beta_{n-1} \geq \cdots \geq \beta_{1} \geq \beta_{0}, \gamma_{n} \geq \gamma_{n-1} \geq \cdots \geq \gamma_{1} \geq$ $\gamma_{0}, \delta_{n} \geq \delta_{n-1} \geq \cdots \geq \delta_{1} \geq \delta_{0}$ lie in:

$$
|q| \leq \frac{1}{\left|a_{n}\right|}\left[\left(\left|\alpha_{0}\right|-\alpha_{0}+\alpha_{n}\right)+\left(\left|\beta_{0}\right|-\beta_{0}+\beta_{n}\right)+\left(\left|\gamma_{0}\right|-\gamma_{0}+\gamma_{n}\right)+\left(\left|\delta_{0}\right|-\delta_{0}+\delta_{n}\right)\right] .
$$

Definition 2.3 [5, Definition 3.1] If $f(q)=\sum_{n=0}^{\infty} q^{n} a_{n}$ and $g(q)=\sum_{n=0}^{\infty} q^{n} b_{n}$ be given power series quaternionic power series with radii of convergence greater than $R$. Then, the real product $f$ and $g$ is defined as $f \star g(q)=\sum_{n=0}^{\infty} q^{n} c_{n}$, where $c_{n}=\sum_{s=0}^{n} a_{s} b_{n-s}$.

\section{Main results}

In this direction, we first prove the following interesting result in which we relax the hypothesis of Theorem 2.1 and, hence, is a generalization of Theorem 2.2. In fact, we prove the following:

Theorem 3.1 All the zeros of the polynomial $p \in \mathbb{P}_{n}$ with quaternionic coefficients $a_{\rho} \in \mathbb{H}, 0 \leq \rho \leq n$ such that:

$$
\begin{aligned}
& \alpha_{n} \geq \alpha_{n-1} \geq \cdots \geq \alpha_{l}, \beta_{n} \geq \beta_{n-1} \geq \cdots \geq \beta_{l}, \\
& \gamma_{n} \geq \gamma_{n-1} \geq \cdots \geq \gamma_{l}, \delta_{n} \geq \delta_{n-1} \geq \cdots \geq \delta_{l}, 0 \leq l \leq n,
\end{aligned}
$$

lie in:

$$
\begin{aligned}
|q| \leq & \frac{1}{\left|a_{n}\right|}\left[\left|\alpha_{0}\right|+\left|\beta_{0}\right|+\left|\gamma_{0}\right|+\left|\delta_{0}\right|+\left(\alpha_{n}-\alpha_{l}\right)+\left(\beta_{n}-\beta_{l}\right)+\left(\gamma_{n}-\gamma_{l}\right)\right. \\
& \left.+\left(\delta_{n}-\delta_{l}\right)+M_{l}\right] .
\end{aligned}
$$

Here:

$$
M_{l}=\sum_{s=1}^{l}\left[\left|\alpha_{s}-\alpha_{s-1}\right|+\left|\beta_{s}-\beta_{s-1}\right|+\left|\gamma_{s}-\gamma_{s-1}\right|+\left|\delta_{s}-\delta_{s-1}\right|\right] .
$$

Applying above Theorem 3.1 for the polynomial $p(q)$ having real coefficient, i.e., $\beta=\gamma=\delta=0$, we have the following result.

Corollary 3.2 All the zeros of the polynomial $p \in \mathbb{P}_{n}$ with real coefficients $a_{\rho}, 0 \leq \rho \leq n$, such that $a_{n} \geq a_{n-1} \geq \cdots \geq a_{l}, 0 \leq l \leq n$, lie in:

$$
|q| \leq \frac{1}{\left|a_{n}\right|}\left(\left|a_{0}\right|+\left(a_{n}-a_{l}\right)+\sum_{m=1}^{l}\left|a_{m}-a_{m-1}\right|\right) .
$$


If we assume $l=n$, then the following result obtains from Corollary 3.2.

Corollary 3.3 All the zeros of the polynomial $p \in \mathbb{P}_{n}$ with real coefficients $a_{l}, 0 \leq l \leq n$, such that $a_{n} \geq$ $a_{n-1} \geq \cdots \geq a_{0}$, lie in

$$
|q| \leq \frac{1}{\left|a_{n}\right|}\left(\left|a_{0}\right|+\sum_{l=1}^{n}\left|a_{l}-a_{l-1}\right|\right) .
$$

Similar result has been obtained by taking $l=n$ in Theorem 3.1 for quaternionic coefficients. Also, we obtain the following result by applying Corollary 3.2 to the polynomial $p(q t)$ for $t>0$.

Corollary 3.4 All the zeros of the polynomial $p \in \mathbb{P}_{n}$ with real coefficients $a_{l}, 0 \leq l \leq n$, such that for $t>0, t^{n} a_{n} \geq t^{n-1} a_{n-1} \geq \cdots \geq t^{l} a_{l}, 0 \leq l \leq n$, lie in:

$$
|q| \leq \frac{1}{\left|a_{n}\right|}\left\{\left(a_{n}-t^{n-l} a_{l}\right)+\sum_{m=0}^{l} \frac{\left|t a_{m}-a_{m-1}\right|}{t^{n-m+1}}\right\}, \quad a_{-1}=0 .
$$

By assuming $\alpha_{\rho} \leq \alpha_{\rho-1}, \beta_{\rho} \leq \beta_{\rho-1}, \gamma_{\rho} \leq \gamma_{\rho-1}, \delta_{\rho} \leq \delta_{\rho-1}$ for $1 \leq \rho \leq l$, we have following application Theorem 3.1.

Corollary 3.5 All the zeros of the polynomial $p \in \mathbb{P}_{n}$ with quaternionic coefficients $a_{l} \in \mathbb{H}, 0 \leq l \leq n$, such that:

$$
\begin{gathered}
\alpha_{n} \geq \alpha_{n-1} \geq \cdots \geq \alpha_{l} \leq \alpha_{l-1} \leq \cdots \leq \alpha_{0}, \beta_{n} \geq \beta_{n-1} \geq \cdots \geq \beta_{l} \leq \beta_{l-1} \leq \cdots \leq \beta_{0}, \\
\gamma_{n} \geq \gamma_{n-1} \geq \cdots \geq \gamma_{l} \leq \gamma_{l-1} \leq \cdots \leq \gamma_{0}, \delta_{n} \geq \delta_{n-1} \geq \cdots \geq \delta_{l} \leq \delta_{l-1} \leq \cdots \leq \delta_{0},
\end{gathered}
$$

lie in:

$$
\begin{aligned}
|q| \leq & \frac{1}{\left|a_{n}\right|}\left[\left|\alpha_{0}\right|+\left|\beta_{0}\right|+\left|\gamma_{0}\right|+\left|\delta_{0}\right|+\left(\alpha_{n}-\alpha_{l}+\alpha_{l-1}+\alpha_{0}\right)+\left(\beta_{n}-\beta_{l}+\beta_{l-1}+\beta_{0}\right)\right. \\
& \left.+\left(\gamma_{n}-\gamma_{l}+\gamma_{l-1}+\gamma_{0}\right)+\left(\delta_{n}-\delta_{l}+\delta_{l-1}+\delta_{0}\right)\right] .
\end{aligned}
$$

Remark 3.6 Like Corollaries 3.2, 3.3 and 3.4, we have some generalizations of Corollary 3.5.

Next, we prove the following result which gives the lower bound for the moduli of zeros of $p(q)$.

Theorem 3.7 If $p \in \mathbb{P}_{n}$ with real coefficients $a_{l}, 0 \leq l \leq n$, such that $a_{n} \geq a_{n-1} \geq \cdots \geq a_{l}, 0 \leq l \leq n$, then $p(q)$ does not vanish in:

$$
|q|<\min \left(1, \frac{\left|a_{0}\right|}{\left|a_{n}\right|+a_{n}-a_{l}-\left|a_{0}\right|+M_{l}}\right)
$$

where $M_{l}=\sum_{s=1}^{l}\left|a_{s}-a_{s-1}\right|$.

For $l=0$, Theorem 3.7 reduces to the following result.

Corollary 3.8 If $p \in \mathbb{P}_{n}$ with real coefficients $a_{l}, 0 \leq l \leq n$, such that $a_{n} \geq a_{n-1} \geq \cdots \geq a_{0}$, then $p(q)$ does not vanish in:

$$
|q|<\frac{\left|a_{0}\right|}{\left|a_{n}\right|+a_{n}-\left|a_{0}\right|}
$$

Finally, we prove the following more general result, which is also a generalization of Theorem 2.2. 
Theorem 3.9 If $p \in \mathbb{P}_{n}$ with quaternionic coefficients $a_{\rho} \in \mathbb{H}, 0 \leq \rho \leq n$, such that:

$$
\begin{aligned}
& \alpha_{n} \geq \alpha_{n-1} \geq \cdots \geq \alpha_{l}, \beta_{n} \geq \beta_{n-1} \geq \cdots \geq \beta_{l}, \\
& \gamma_{n} \geq \gamma_{n-1} \geq \cdots \geq \gamma_{l}, \delta_{n} \geq \delta_{n-1} \geq \cdots \geq \delta_{l}, 0 \leq l \leq n,
\end{aligned}
$$

and

$$
\max _{|q|=1}\left|\sum_{s=1}^{l} q^{s}\left(a_{s}-a_{s-1}\right)\right| \leq M,
$$

then all the zeros of $p(q)$ lie in:

$$
\begin{aligned}
|q| \leq & \max \left(1, \frac{1}{\left|a_{n}\right|}\left[\left|\alpha_{0}\right|+\left|\beta_{0}\right|+\left|\gamma_{0}\right|+\left|\delta_{0}\right|+\left(\alpha_{n}-\alpha_{l}\right)+\left(\beta_{n}-\beta_{l}\right)\right.\right. \\
& \left.\left.+\left(\gamma_{n}-\gamma_{l}\right)+\left(\delta_{n}-\delta_{l}\right)+M\right]\right) .
\end{aligned}
$$

Remark 3.10 From Lemma 4.2, $q \in \mathbb{H}$ can be expressed as $q=A e^{B j}$, where $A=a+i b, B=c+i d$, where $a, b, c, d$ are real. If we take $A=\frac{1}{\sqrt{2}}+i \frac{1}{\sqrt{2}}, B=\pi / 4$, then $q=A e^{B j}=\frac{1}{2}(1+i+j+k) \Longrightarrow|q|=1$ and for $|q|=1$ :

$$
\begin{aligned}
M & =\left|\sum_{s=1}^{l} q^{s}\left(a_{s}-a_{s-1}\right)\right| \\
& \leq \sum_{s=1}^{l}\left|a_{s}-a_{s-1}\right||q|^{s} \\
& \leq \sum_{s=1}^{l}\left(\left|\alpha_{s}-\alpha_{s-1}\right|+\left|\beta_{s}-\beta_{s-1}\right|+\left|\gamma_{s}-\gamma_{s-1}\right|+\left|\delta_{s}-\delta_{s-1}\right|\right)|q|^{s} \\
& \leq M_{l}, \text { for }|q|=1 .
\end{aligned}
$$

Here, $M_{l}, 0 \leq l \leq n$ is defined in Theorem 3.1. Therefore, $M \leq M_{l}, 0 \leq l \leq n$. So, we conclude that Theorem 3.9 is a refinement of Theorem 3.1.

The following result is an immediate consequence of the Theorem 3.9 by taking $l=n$.

Corollary 3.11 If $p \in \mathbb{P}_{n}$ with quaternionic coefficients $a_{l} \in \mathbb{H}, 0 \leq l \leq n$, then all the zeros of $p(q)$ lie in:

$$
|q| \leq \frac{M+\left|\alpha_{0}\right|+\left|\beta_{0}\right|+\left|\gamma_{0}\right|+\left|\delta_{0}\right|}{\left|a_{n}\right|}
$$

where

$$
M=\max _{|q|=1}\left|\sum_{s=1}^{n} q^{s}\left(a_{s}-a_{s-1}\right)\right| .
$$

\section{Lemma}

Lemma 4.1 [5, Theorem 3.3] Let $f(q)=\sum_{n=0}^{\infty} q^{n} a_{n}$ and $g(q)=\sum_{n=0}^{\infty} q^{n} b_{n}$ be given quaternionic power series with radii of convergence greater than $R$. The real product of $f(q)$ and $g(q)$ is defined as $(f \star g)(q)=$ $\sum_{n=0}^{\infty} q^{n} c_{n}$, where $c_{n}=\sum_{s=0}^{n} a_{s} b_{n-s}$. Let $\left|q_{0}\right|<R$. Then, $(f \star g)\left(q_{0}\right)=0$ if and only if $f\left(q_{0}\right)=0$ or $f\left(q_{0}\right) \neq 0$ implies $g\left(f\left(q_{0}\right)^{-1} q_{0} f\left(q_{0}\right)\right)=0$.

Lemma 4.2 [11] Every quaternion $q=\alpha+i \beta+j \gamma+k \delta \in \mathbb{H}$, where $\alpha, \beta, \gamma, \delta$ are real, can be expressed in form $q=A e^{B j}$, where $A=a+i b$ and $B=c+j d$. 


\section{Proof of statements}

Proof of Theorem 3.1 Consider the polynomial:

$$
f(q)=\sum_{s=1}^{n} q^{l}\left(a_{s}-a_{s-1}\right)+a_{0}
$$

and $p(q) \star(1-q)=f(q)-q^{n+1} a_{n}$. From Lemma 4.1, $p(q) \star(1-q)=0$ if and only if either $p(q)=0$, or $p(q) \neq 0$ implies $p(q)^{-1} q p(q)-1=0$, i.e., $p(q)^{-1} q p(q)=1$. If $p(q) \neq 1$, then $q=1$. Therefore, the only zeros of $p(q) \star(1-q)$ are $q=1$ and the zeros of $p(q)$. For $|q|=1$ :

$$
\begin{aligned}
|f(q)| \leq & \left|a_{0}\right|+\sum_{s=1}^{n}\left|a_{s}-a_{s-1}\right| \\
= & \left|\alpha_{0}+i \beta_{0}+j \gamma_{0}+k \delta_{0}\right|+\sum_{s=1}^{n} \mid\left(\alpha_{s}-\alpha_{s-1}\right)+i\left(\beta_{s}-\beta_{s-1}\right)+j\left(\gamma_{s}-\gamma_{s-1}\right) \\
& +k\left(\delta_{s}-\delta_{s-1}\right) \mid \\
\leq & \left|\alpha_{0}\right|+\left|\beta_{0}\right|+\left|\gamma_{0}\right|+\left|\delta_{0}\right|+\sum_{s=1}^{n}\left[\left|\alpha_{s}-\alpha_{s-1}\right|+\left|\beta_{s}-\beta_{s-1}\right|+\left|\gamma_{s}-\gamma_{s-1}\right|\right. \\
& \left.+\left|\delta_{s}-\delta_{s-1}\right|\right] \\
\leq & \left|\alpha_{0}\right|+\left|\beta_{0}\right|+\left|\gamma_{0}\right|+\left|\delta_{0}\right|+\left(\alpha_{n}-\alpha_{l}\right)+\left(\beta_{n}-\beta_{l}\right)+\left(\gamma_{n}-\gamma_{l}\right)+\left(\delta_{n}-\delta_{l}\right) \\
& +\sum_{s=1}^{l}\left[\left|\alpha_{s}-\alpha_{s-1}\right|+\left|\beta_{s}-\beta_{s-1}\right|+\left|\gamma_{s}-\gamma_{s-1}\right|+\left|\delta_{s}-\delta_{s-1}\right|\right] .
\end{aligned}
$$

Since

$$
\max _{|q|=1}\left|q^{n} \star f(1 / q)\right|=\max _{|q|=1}|f(1 / q)|=\max _{|q|=1}|f(q)| ;
$$

therefore, $q^{n} \star f(1 / q)$ have same bound on $|q|=1$ as $f(q)$, that is:

$$
\begin{aligned}
\left|q^{n} \star f(1 / q)\right| \leq & \left|\alpha_{0}\right|+\left|\beta_{0}\right|+\left|\gamma_{0}\right|+\left|\delta_{0}\right|+\left(\alpha_{n}-\alpha_{l}\right)+\left(\beta_{n}-\beta_{l}\right)+\left(\gamma_{n}-\gamma_{l}\right) \\
& +\left(\delta_{n}-\delta_{l}\right)+M_{l},
\end{aligned}
$$

for $|q|=1$. Where $M_{l}=\sum_{s=1}^{l}\left[\left|\alpha_{s}-\alpha_{s-1}\right|+\left|\beta_{s}-\beta_{s-1}\right|+\left|\gamma_{s}-\gamma_{s-1}\right|+\left|\delta_{s}-\delta_{s-1}\right|\right.$. Then, by maximum modulus theorem [6, Theorem 3.4]:

$$
\begin{aligned}
\left|q^{n} \star f(1 / q)\right|= & \left|q^{n} f(1 / q)\right| \leq\left|\alpha_{0}\right|+\left|\beta_{0}\right|+\left|\gamma_{0}\right|+\left|\delta_{0}\right|+\left(\alpha_{n}-\alpha_{l}\right)+\left(\beta_{n}-\beta_{l}\right) \\
& +\left(\gamma_{n}-\gamma_{l}\right)+\left(\delta_{n}-\delta_{l}\right)+M_{l},
\end{aligned}
$$

that is:

$$
\begin{aligned}
|f(1 / q)| \leq & \frac{1}{|q|^{n}}\left[\left|\alpha_{0}\right|+\left|\beta_{0}\right|+\left|\gamma_{0}\right|+\left|\delta_{0}\right|+\left(\alpha_{n}-\alpha_{l}\right)+\left(\beta_{n}-\beta_{l}\right)+\left(\gamma_{n}-\gamma_{l}\right)\right. \\
& \left.+\left(\delta_{n}-\delta_{l}\right)+M_{l}\right],
\end{aligned}
$$

for $|q| \leq 1$. Replacing $q$ by $1 / q$, we have for $|q| \geq 1$ :

$$
\begin{aligned}
|f(q)| \leq & {\left[\left|\alpha_{0}\right|+\left|\beta_{0}\right|+\left|\gamma_{0}\right|+\left|\delta_{0}\right|+\left(\alpha_{n}-\alpha_{l}\right)+\left(\beta_{n}-\beta_{l}\right)+\left(\gamma_{n}-\gamma_{l}\right)\right.} \\
& \left.+\left(\delta_{n}-\delta_{l}\right)+M_{l}\right]|q|^{n} .
\end{aligned}
$$

Also:

$$
\begin{aligned}
|p(q) \star(1-q)| & =\left|f(q)-q^{n+1} a_{n}\right| \\
& \geq\left|a_{n}\right||q|^{n+1}-|f(q)| .
\end{aligned}
$$


On using (5.1), we have for $|q| \geq 1$ :

$$
\begin{aligned}
|p(q) \star(1-q)| \geq & {\left[\left|a_{n}\right||q|-\left\{\left|\alpha_{0}\right|+\left|\beta_{0}\right|+\left|\gamma_{0}\right|+\left|\delta_{0}\right|+\left(\alpha_{n}-\alpha_{l}\right)+\left(\beta_{n}-\beta_{l}\right)\right.\right.} \\
& \left.\left.+\left(\gamma_{n}-\gamma_{l}\right)+\left(\delta_{n}-\delta_{l}\right)+M_{l}\right\}\right]|q|^{n} .
\end{aligned}
$$

This implies that $|p(q) \star(1-q)|>0$, i.e., $p(q) \star(1-q) \neq 0$ if:

$$
\begin{aligned}
|q|> & \frac{1}{\left|a_{n}\right|}\left[\left|\alpha_{0}\right|+\left|\beta_{0}\right|+\left|\gamma_{0}\right|+\left|\delta_{0}\right|+\left(\alpha_{n}-\alpha_{l}\right)+\left(\beta_{n}-\beta_{l}\right)+\left(\gamma_{n}-\gamma_{l}\right)\right. \\
& \left.+\left(\delta_{n}-\delta_{l}\right)+M_{l}\right] .
\end{aligned}
$$

Since the only zeros $p(q) \star(1-q)$ are $q=1$ and the zeros of $p(q)$. Then, $p(q) \neq 0$ for:

$$
\begin{aligned}
|q|> & \frac{1}{\left|a_{n}\right|}\left[\left|\alpha_{0}\right|+\left|\beta_{0}\right|+\left|\gamma_{0}\right|+\left|\delta_{0}\right|+\left(\alpha_{n}-\alpha_{l}\right)+\left(\beta_{n}-\beta_{l}\right)+\left(\gamma_{n}-\gamma_{l}\right)\right. \\
& \left.+\left(\delta_{n}-\delta_{l}\right)+M_{l}\right] .
\end{aligned}
$$

Hence, all the zeros of $p(q)$ lie in:

$$
\begin{aligned}
|q| \leq & \frac{1}{\left|a_{n}\right|}\left[\left|\alpha_{0}\right|+\left|\beta_{0}\right|+\left|\gamma_{0}\right|+\left|\delta_{0}\right|+\left(\alpha_{n}-\alpha_{l}\right)+\left(\beta_{n}-\beta_{l}\right)+\left(\gamma_{n}-\gamma_{l}\right)\right. \\
& \left.+\left(\delta_{n}-\delta_{l}\right)+M_{l}\right] .
\end{aligned}
$$

This proves Theorem 3.1.

Proof of Theorem 3.7 Consider the reciprocal polynomial:

$$
R(q)=q^{n} \star p(1 / q)=\sum_{l=0}^{n} q^{n-l} a_{l} .
$$

Let $R(q) \star(1-q)=g(q)-q^{n+1} a_{0}$, where $g(q)=\sum_{s=1}^{n} q^{n-s+1}\left(a_{s-1}-a_{s}\right)+a_{n}$. Now:

$$
|g(q)| \leq \sum_{s=1}^{n}|q|^{n-s+1}\left|a_{s-1}-a_{s}\right|+\left|a_{n}\right|
$$

For $|q|=1$, we have:

$$
\begin{aligned}
|g(q)| & \leq \sum_{s=1}^{n}\left|a_{s-1}-a_{s}\right|+\left|a_{n}\right| \\
& \leq M_{l}+\left(a_{n}-a_{l}+\left|a_{n}\right|\right) .
\end{aligned}
$$

Using the same argument as in proof Theorem 3.1, we have for $|q|>1$ :

$$
|g(q)| \leq\left[M_{l}+a_{n}-a_{l}+\left|a_{n}\right|\right]|q|^{n} .
$$

This implies that, for $|q|>1$ :

$$
\begin{aligned}
|R(q) \star(1-q)| & =\left|g(q)-q^{n+1} a_{0}\right| \geq\left|a_{0}\right||q|^{n+1}-|g(q)| \\
& \geq\left[\left|a_{0}\right||q|-\left(M_{l}+a_{n}-a_{l}+\left|a_{n}\right|\right)\right]|q|^{n}>0,
\end{aligned}
$$

if

$$
|q|>\frac{M_{l}+a_{n}-a_{l}+\left|a_{n}\right|}{\left|a_{0}\right|},
$$

i.e $R(q) \star(1-q) \neq 0$ for $|q|>\frac{M_{l}+a_{n}-a_{l}+\left|a_{n}\right|}{\left|a_{0}\right|}$. Hence, all the zeros of $R(q) \star(1-q)$ whose modulus greater than 1 lie in:

$$
|q| \leq \frac{M_{l}+a_{n}-a_{l}+\left|a_{n}\right|}{\left|a_{0}\right|},
$$


i.e., all the zeros of $R(q)$ lie in:

$$
|q| \leq \max \left(1, \frac{M_{l}+a_{n}-a_{l}+\left|a_{n}\right|}{\left|a_{0}\right|}\right) .
$$

Therefore, all the zeros $p(q)$ lie in

$$
|q| \geq \min \left(1, \frac{\left|a_{0}\right|}{M_{l}+a_{n}-a_{l}+\left|a_{n}\right|}\right) .
$$

Thus, the polynomial does not vanish in

$$
|q|<\min \left(1, \frac{\left|a_{0}\right|}{M_{l}+a_{n}-a_{l}+\left|a_{n}\right|}\right) .
$$

This completes the proof of Theorem 3.7.

Proof of Theorem 3.9 Define:

$$
f(q)=\sum_{s=1}^{n} q^{s}\left(a_{s}-a_{s-1}\right)+a_{0},
$$

such that $p(q) \star(1-q)=f(q)-q^{n+1} a_{n}$. Let:

$$
R(q)=q^{n} \star f(1 / q)=\sum_{s=1}^{n} q^{n-s}\left(a_{s}-a_{s-1}\right)+q^{n} a_{0} .
$$

Then, we have:

$$
\begin{aligned}
|R(q)| \leq & \left|\sum_{s=1}^{n} q^{n-s}\left(a_{s}-a_{s-1}\right)\right|+|q|^{n}\left|a_{0}\right| \\
\leq & |q|^{n}\left[\left|\alpha_{0}\right|+\left|\beta_{0}\right|+\left|\gamma_{0}\right|+\left|\delta_{0}\right|\right]+\left|\sum_{s=1}^{l} q^{n-s}\left(a_{s}-a_{s-1}\right)\right| \\
& +\left|\sum_{s=l+1}^{n} q^{n-s}\left(a_{s}-a_{s-1}\right)\right| \\
\leq & |q|^{n}\left[\left|\alpha_{0}\right|+\left|\beta_{0}\right|+\left|\gamma_{0}\right|+\left|\delta_{0}\right|\right]+\left.\left|\sum_{s=1}^{l}\right| q\right|^{n-s}\left(a_{s}-\alpha_{s-1}\right) \mid \\
& +\sum_{s=l+1}^{n}|q|^{n-s}\left[\left|a_{s}-\alpha_{s-1}\right|+\left|\beta_{s}-\beta_{s-1}\right|+\left|\gamma_{s}-\gamma_{s-1}\right|+\left|\delta_{s}-\delta_{s-1}\right|\right]
\end{aligned}
$$

For $|q|=1$ :

$$
\begin{aligned}
|R(q)| \leq & M+\left|\alpha_{0}\right|+\left|\beta_{0}\right|+\left|\gamma_{0}\right|+\left|\delta_{0}\right| \\
& +\sum_{s=l+1}^{n}\left[\left|a_{s}-\alpha_{s-1}\right|+\left|\beta_{s}-\beta_{s-1}\right|+\left|\gamma_{s}-\gamma_{s-1}\right|+\left|\delta_{s}-\delta_{s-1}\right|\right] \\
\leq & M+\left|\alpha_{0}\right|+\left|\beta_{0}\right|+\left|\gamma_{0}\right|+\left|\delta_{0}\right|+\left(\alpha_{n}-\alpha_{l}\right)+\left(\beta_{n}-\beta_{l}\right)+\left(\gamma_{n}-\gamma_{l}\right)+\left(\delta_{n}-\delta_{l}\right),
\end{aligned}
$$

where $M=\max _{|q|=1}\left|\sum_{s=1}^{l} q^{s}\left(a_{s}-a_{s-1}\right)\right|$. Hence, using the same argument as in Proof of Theorem 3.1, we have for $|q| \geq 1$ :

$$
\begin{aligned}
f(q) \leq & |q|^{n}\left[M+\left|\alpha_{0}\right|+\left|\beta_{0}\right|+\left|\gamma_{0}\right|+\left|\delta_{0}\right|+\left(\alpha_{n}-\alpha_{l}\right)+\left(\beta_{n}-\beta_{l}\right)\right. \\
& \left.+\left(\gamma_{n}-\gamma_{l}\right)+\left(\delta_{n}-\delta_{l}\right)\right] .
\end{aligned}
$$


Now:

$$
p(q) \star(1-q)=f(q)-q^{n+1} a_{n},
$$

that is:

$$
|p(q) \star(1-q)| \geq\left.|| a_{n}|| q\right|^{n+1}-|f(q)| .
$$

From 5.4:

$$
\begin{aligned}
|p(q) \star(1-q)| \geq & {\left[\left|a_{n}\right||q|-\left(M+\left|\alpha_{0}\right|+\left|\beta_{0}\right|+\left|\gamma_{0}\right|+\left|\delta_{0}\right|+\left(\alpha_{n}-\alpha_{k}\right)+\left(\beta_{n}-\beta_{k}\right)\right.\right.} \\
& \left.\left.+\left(\gamma_{n}-\gamma_{k}\right)+\left(\delta_{n}-\delta_{k}\right)\right)\right]|q|^{n} \text { for }|q|>1 .
\end{aligned}
$$

This gives all the zeros of $p(q) \star(1-q)$, whose modulus is greater than 1 lie in:

$$
|q| \leq \frac{M+\left|\alpha_{0}\right|+\left|\beta_{0}\right|+\left|\gamma_{0}\right|+\left|\delta_{0}\right|+\left(\alpha_{n}-\alpha_{l}\right)+\left(\beta_{n}-\beta_{l}\right)+\left(\gamma_{n}-\gamma_{l}\right)+\left(\delta_{n}-\delta_{l}\right)}{\left|a_{n}\right|} .
$$

Thus, all the zeros of $p(q)$ lie in:

$$
\begin{aligned}
|q| \leq & \max \left(1, \frac{1}{\left|a_{n}\right|}\left[\left|\alpha_{0}\right|+\left|\beta_{0}\right|+\left|\gamma_{0}\right|+\left|\delta_{0}\right|+\left(\alpha_{n}-\alpha_{l}\right)+\left(\beta_{n}-\beta_{l}\right)\right.\right. \\
& \left.\left.+\left(\gamma_{n}-\gamma_{l}\right)+\left(\delta_{n}-\delta_{l}\right)+M\right]\right) .
\end{aligned}
$$

The proof of Theorem (3.9) is now complete.

Acknowledgements The author would like to thank the referees for their valuable suggestions which helped to improve the quality of this work.

Open Access This article is licensed under a Creative Commons Attribution 4.0 International License, which permits use, sharing, adaptation, distribution and reproduction in any medium or format, as long as you give appropriate credit to the original author(s) and the source, provide a link to the Creative Commons licence, and indicate if changes were made. The images or other third party material in this article are included in the article's Creative Commons licence, unless indicated otherwise in a credit line to the material. If material is not included in the article's Creative Commons licence and your intended use is not permitted by statutory regulation or exceeds the permitted use, you will need to obtain permission directly from the copyright holder. To view a copy of this licence, visit http://creativecommons.org/licenses/by/4.0/.

\section{References}

1. Aziz, A.; Zargar, B.A.: Some extension of Eneström-Kakeya theorem. Glasńik Mathematiki. 31, 239-244 (1996)

2. Carney, N., Gardner, R., Keaton, R. and Powers, A.: The Eeström-Kakeya theorem of a quaternionic variable. J. Appl. Theory, 250, Article 105325 (2020)

3. Eneström, G.: Remarque sur un théoréme relatif aux racines de l'équation $a_{n} x^{n}+\cdots+a_{0}=0$ oú tous les coefficients sont réels et positifs, Tohoku Math. J. 18, 34-36 (1920)

4. Govil, N.K.; Rahman, Q.I.: On the Eneström-Kakeya theorem. Tohoku Math. J. 20(2), 126-136 (1968)

5. Gentili, G.; Stoppato, C.: Zeros of regular functions and polynomials of a quaternionic variable. Michigan Math. J. 56, 655-667 (2008)

6. Gentili, G.; Struppa, : A new theory of regular functions of a quaternionic variable. Adv. Math. 216, 279-301 (2007)

7. Hurwitz, A.: Über einen Satz des Harrn Kakeya. Tohoku Math. J. First Ser. 4, 626-631 (1913-1914)

8. Kakeya, S.: On the limits of the roots of an algebraic equation with positive coefficient. Tohoku Math. J. 2, 140-142 (1912-1913)

9. Joyal, A.; Labelle, G.; Rahman, Q.I.: On the location of zeros of polynomials. Can. Math. Bull. 10(1), 53-63 (1967)

10. Marden, M.: Geometry of Polynomials. Math. Surveys, No. 3, Amer. Math. Soc.

11. Sangwine, S.J.; Bihan, N.L.: Quaternion polar representation with a complex modulus and complex argument inspired by the Cayley-Dickson. Adv. Appl. Cliffoard Algebra 20(1), 111-120 (2010)

Publisher's Note Springer Nature remains neutral with regard to jurisdictional claims in published maps and institutional affiliations. 